\title{
Solution of nonlinear curvature driven evolution of plane convex curves
}

\author{
Karol Mikula ${ }^{1}$ \\ Slovak Technical University, Department of Mathematics, Radlinskeho 11, 81368 Bratislava, Slovakia
}

\begin{abstract}
The numerical approximation scheme for solving the nonlinear initial value problem

$$
\partial_{t} b(v)=\partial_{x x} \beta(x, v)+\beta(x, v)
$$

with periodic boundary conditions is presented. Local existence and uniqueness of a solution and convergence of approximations is a consequence of the results of Mikula and Kačur (1996), where the anisotropic curvature driven evolution of plane convex curves is studied. The considered problem is a nonlinear generalization of plane convex curves evolution depending on curvature, known as curve shortening flow. It corresponds to the evolution equation
\end{abstract}

$$
\nu=\beta(\theta, k),
$$

where $\nu$ is the normal velocity of the curve, $k$ its curvature and $\theta$ the angle of the tangent to the curve with horizontal axis. It arises in the theory of image and shape multiscale analysis introduced by Alvarez, Guichard, Lions and Morel and Sapiro and Tannenbaum, and also in anisotropic interface motions proposed by Angenent and Gurtin. () 1997 Elsevier Science B.V.

Keywords: Mean curvature flow; Curve shortening; Image and shape multiscale analysis; Phase interface; Nonlinear degenerate parabolic equations; Blow-up; Numerical solution

\section{Introduction and approximation scheme}

Evolution of plane convex curves, which depends in a nonlinear and anisotropic way on curvature, can be modeled by the following double-nonlinear initial-boundary value problem. Let $v(x, t)$ be a smooth function which satisfies

$$
\begin{aligned}
& \partial_{t} b(v)=\partial_{x x} \beta(x, v)+\beta(x, v), \\
& v(x, t)=v(x+2 \pi \nu, t) \\
& v(x, 0)=v_{0}(x)
\end{aligned}
$$

\footnotetext{
${ }^{1}$ E-mail: mikula@ops.svf.stuba.sk. 
for $x \in \mathbb{R}$ and $t \in[0, T)$, where $\nu \in \mathbb{N}, v_{0}$ is an initial function and

$$
\begin{aligned}
& b(s) \in C^{2}(-\infty, a), a<\infty, \text { satisfying } b^{\prime}(s)>0, \\
& b(s) \rightarrow a_{1} \quad \text { and } \quad b^{\prime}(s) \rightarrow 0 \quad \text { for } s \rightarrow-\infty, \\
& b(s) \rightarrow \infty \quad \text { and } \quad b^{\prime}(s) \rightarrow \infty \quad \text { for } s \rightarrow a^{-}, \\
& \beta(x, s)=S(x) \gamma(s)+U(x),
\end{aligned}
$$

$S \geqslant q>0, S_{x}, S_{x x}, U$ are bounded measurable functions, periodic in interval $[0,2 \pi \nu]$,

$$
\gamma \text { is } C^{2} \text {-function in } \mathbb{R}-\{a\} \text { with } \gamma^{\prime}(s)>0, s \neq a, \gamma^{\prime}(a) \geqslant 0,
$$

$$
\gamma(a)=a \text {. }
$$

The nonlinearities are represented by the increasing functions $b(s), \beta(x, s)$ admitting asymptotical degeneracies expressed by the hypotheses $(\mathrm{H} 2)-(\mathrm{H} 4)$. The special form of the problem causes a possible blow-up of the solution in a finite time. Asymptotical degeneracy of "slow diffusion type" $\left(b^{\prime}=\infty, \gamma^{\prime}=0\right)$ is related to the parts of the curves where curvature is close to 0 and plays a role in the presence of anisotropy and porous-medium-like nonlinearity (e.g., $\gamma(s)=s^{m}, m>1$ ). Asymptotical degeneracy of "fast diffusion type" $\left(b^{\prime}=0, \gamma^{\prime}=\infty\right)$ is related to both large and small curvatures, and plays a role near the shrinking, singularities formation and influences a more shape preserving evolution (see the numerical experiments of Section 2).

Provided $b(s)=-1 / s$ and in the case of initial closed and convex plane curve, the problem (1.1) is related to the curve evolution equation

$$
\mathcal{V}=\beta(\theta, k),
$$

where $\mathcal{V} \equiv \mathcal{V}(Q, t)$ is the normal velocity, $k(Q, t)$ the curvature of the curve and $\theta(Q, t)$ the angle of tangent to the curve with horizontal axis at a point $Q$ and time $t$.

Let the initial curve $\boldsymbol{r}_{0}$ be parameterized by $\theta$. Let $k_{0}(\theta)$ be its curvature and $\nu \in \mathbb{N}$ be its index. Following the lines of Gage and Hamilton [14] or using the results of Angenent and Gurtin [7, (2.23)] we have that the flow $\boldsymbol{r}(\theta, t)$ of the curves, which solves the problem (1.2) with the initial curve $\boldsymbol{r}_{0}$, is given uniquely up to translation by the formula

$$
\boldsymbol{r}\left(\theta_{0}, t\right)=\boldsymbol{r}(0, t)-\int_{0}^{\theta_{0}} \frac{\mathrm{e}^{\mathrm{i} \theta}}{k(\theta, t)} \mathrm{d} \theta,
$$

in which the curvature $k(\theta, t)$ is the solution of (1.1) with initial condition given by $k_{0}(\theta)$. Note here, that by convention introduced in [7], the curvature of strictly convex curves is negative.

Geometrical equations like (1.2) describe several phenomena in physics, material sciences, computer vision and artificial intelligence. There are two main fields, where the curve motions play an important role. First, there is the multiscale analysis of images and shapes, which is closely related to signal smoothing, edge detection and image representation (see, e.g., $[2,4,22])$. The second is the Stefan problem with surface tension and related anisotropic interface motion models (see, e.g., [7,8,25,29]). 

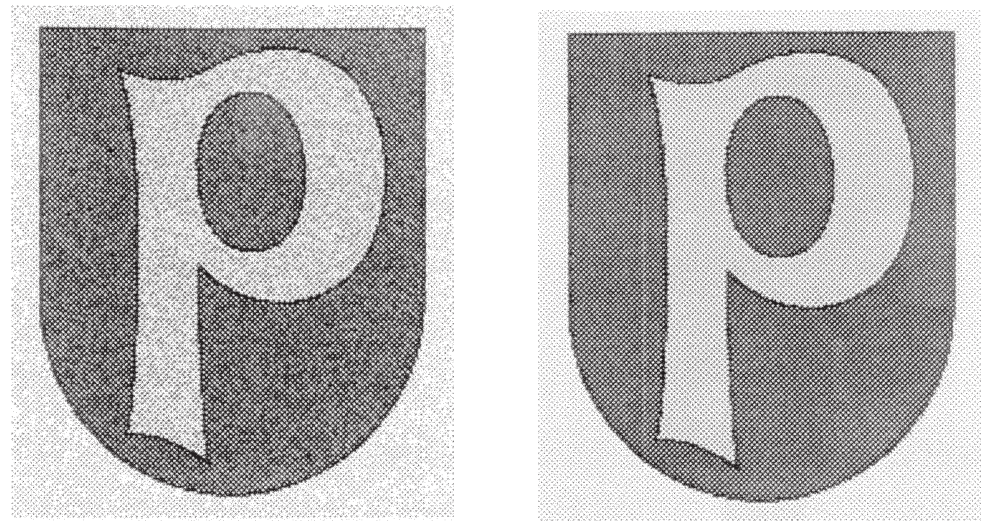

Fig. 1. The scanned emblem—Podolinec (left) and its smoothed version (right).
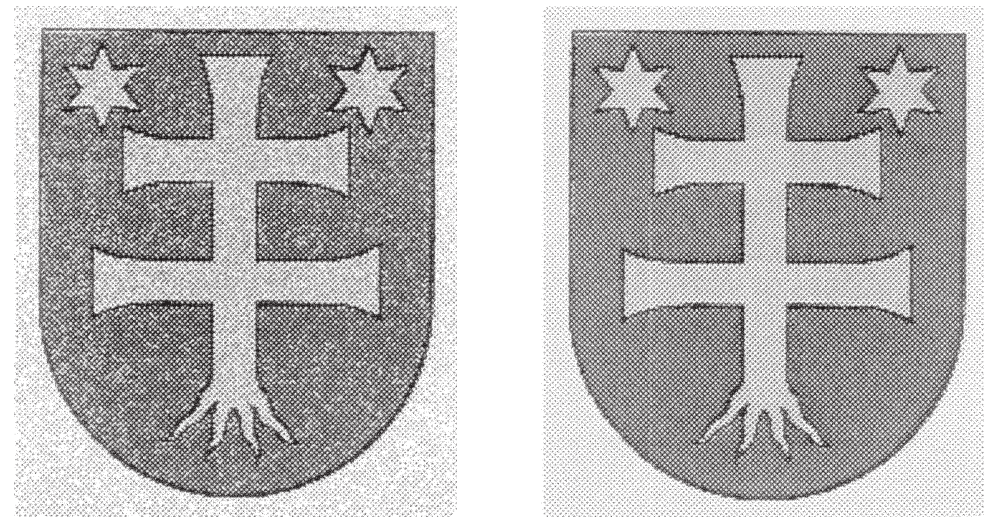

Fig. 2. The scanned emblem-Žilina (left) and its smoothed version (right).

In the context of image processing, the so-called morphological image multiscale analysis is widely used. This analysis is represented by a viscosity solution $[10,11,13]$ of the following nonlinear degenerate parabolic equation in a two-dimensional rectangular domain

$$
u_{t}=|\nabla u| F(\operatorname{div}(\nabla u /|\nabla u|)),
$$

where $F$ is a nondecreasing function [2,4,23]. The initial condition corresponds to the processed image and the solution $u$ to its scaling version ( $t$ is understood as an abstract parameter-scale). The classical mean curvature equation is obtained in the case $F(s)=s$. The silhouette (boundary of a shape) in the image corresponds to a level line of $u$. Eq. (1.4) leads to the evolution equation for image level lines (see, e.g., [4]):

$$
\mathcal{V}=F(k) \text {. }
$$

The scaling of silhouettes by Eq. (1.5) is called morphological shape multiscale analysis. In the vision theory, affine invariant multiscale space, which is a natural generalization of linear mean curvature motion and is given by $F(k)=k^{1 / 3}$, is of special interest (see, e.g., [2,28]). We present Figs. 1 
and 2 for a demonstration of the practical usage of models like (1.4) in image processing. The ancient emblems of two Slovak towns (Podolinec and Žilina) are scanned from the book with neither paper nor colors of good quality. The damage of the images is removed-smoothed versions of emblems are obtained by solving numerically [16] a certain regularization of the PDE

$$
u_{t}=q(|\nabla u|)|\nabla u| \operatorname{div}(\nabla u /|\nabla u|),
$$

with zero Neumann boundary conditions and with an initial condition given by the scanned picture. The choice of the threshold function $q(s)$ equals, e.g., $1 /\left(1+s^{2}\right)$, causes stopping of the motion of silhouettes on which the gradient is large (i.e., important edges) and of classical curvature driven motion of other level lines of image, which implies the smoothing of required regions [3].

In the context of multiphase thermomechanics with interfacial structure, there arises the curve evolution model

$$
h(\theta) \mathcal{V}=g(\theta) k-f,
$$

which describes anisotropic motion of phase interfaces. The functions $h, g, f$ come from the constitutive description of the interface and represent physical quantities such as kinetic coefficient, interfacial free energy and energy difference of phases, respectively (see, e.g., [7,8]). Dependence on $\theta$ yields the anisotropy to the model. Interesting properties of isotropic linear curve shortening flow are given in $[1,5,14,15]$. The general model (1.2) was studied analytically in [6] in the case of evolution on arbitrary surfaces.

The main goal of this paper is to apply the computational method developed in [25] to a new class of geometrical equations (1.2) arising recently in the theory of image and shape analysis. We adapt an existing numerical approximation [25, (2.8)-(2.10)], successful for the case $\gamma(s)=s$, to the double-nonlinear case (1.1).

If we denote $I=(0, T), \Omega=(0,2 \pi \nu), Q_{T}=I \times \Omega, V=\left\{w \in W_{2}^{1}(\Omega): w(0)=w(2 \pi \nu)\right\}, V^{*}$ its dual space, and assume that $\gamma\left(v_{0}\right) \in V$, we can define the weak solution of (1.1) as a function $v \in L_{2}\left(I, L_{2}\right)$ with $\partial_{t} b(v) \in L_{2}\left(I, V^{*}\right)$ for which $\beta(x, v) \in L_{2}(I, V), v(0)=v_{0}$ and

$$
\int_{I}\left\langle\partial_{t} b(v), \varphi\right\rangle+\int_{I}\left(\partial_{x} \beta(x, v), \varphi_{x}\right)=\int_{I}(\beta(x, v), \varphi), \quad \forall \varphi \in L_{2}(I, V) .
$$

Defining

$$
\begin{aligned}
& \widetilde{b}(s):=b\left(\gamma^{-1}(s)\right), \\
& \widetilde{\beta}(x, s):=\beta\left(x, \gamma^{-1}(s)\right)=S(x) s+U(x),
\end{aligned}
$$

we can transform (1.1) into the form

$$
\begin{aligned}
\partial_{t} \widetilde{b}(w) & =\partial_{x x} \widetilde{\beta}(x, w)+\widetilde{\beta}(x, w), \\
w(x, t) & =w(x+2 \pi \nu, t), \\
w(x, 0) & =w_{0}(x)
\end{aligned}
$$

which is included in the framework of the model equation

$$
\partial_{t} \widetilde{b}(v)=\left(A(x) v_{x}\right)_{x}+(B(x) v)_{x}+D(x) v+G(x)
$$


studied numerically in [25]. Following the idea of [25], we can regularize (1.8) by considering globally Lipschitz continuous function $\widetilde{b}_{R}(s)$ instead of $\widetilde{b}(s)$. The regularization is chosen so that $\widetilde{b}_{R}$ and $\widetilde{b}$ are the same for arguments in the interval $(-R, a-1 / R)$, where $R$ is a (large) regularization parameter (let us denote $\lambda=\widetilde{b}^{\prime}(-R)>0$ ). Theorem 2.1 of [25] guarantees existence and uniqueness of the weak solution $w$ of the regularized (1.8) problem. Theorem 2.2 of [25] then transfers the results locally in time to the unregularized problem (1.8), and due to the properties of $\gamma$ also to the original problem (1.1). We briefly recall the idea. Let $w(x, 0) \in L_{\infty}(\Omega) \cap V$ and

$$
-R+\delta<w(x, 0)<a-\frac{1}{R}-\delta .
$$

Then we have obtained $L_{\infty}$-estimates for $w$ and $\widetilde{b}_{R}(w)$, which imply the existence of $0<T_{1}<\infty$ such that

$$
-R \leqslant w(x, t) \leqslant a-\frac{1}{R}
$$

hold for every $t \in\left(0, T_{1}\right)$, a.e. $x \in \Omega$ (see [25, Theorem 2.2]). The functions $\widetilde{b}_{R}$ and $\widetilde{b}$ are the same for the arguments satisfying (1.9), so the solutions of regularized and unregularized problems are the same in time interval $\left(0, T_{1}\right)$.

Let us solve numerically the problem (1.1) in the time interval $(0, T)$ (where $T=T_{1}$ ). Note that, if the regularization parameter $R$ is chosen sufficiently large, $T$ can be rather near to the blow-up time of curvature. We use the following approximation.

\section{Approximation scheme}

Let $n \in \mathbb{N}, \tau=T / n, t_{i}=i \tau$ for $i=0, \ldots, n, v_{0}=v_{0}(x), u_{0}=\gamma\left(v_{0}\right)$. For $i=1, \ldots, n$ we look for functions $u_{i} \in V\left(u_{i} \approx \gamma\left(v_{i}\right)\right), \quad \mu_{i} \in L_{\infty}(\Omega)$ such that

$$
\left(\mu_{i}\left(u_{i}-\gamma\left(v_{i-1}\right)\right), \varphi\right)+\tau\left(\partial_{x} \widetilde{\beta}\left(x, u_{i}\right), \varphi_{x}\right)=\tau\left(\widetilde{\beta}\left(x, u_{i}\right), \varphi\right), \quad \forall \varphi \in V,
$$

provided the "convergence condition"

$$
\alpha \frac{\lambda}{2} \leqslant \mu_{i} \leqslant \frac{b\left(\gamma^{-1}\left(\alpha u_{i}+(1-\alpha) \gamma\left(v_{i-1}\right)\right)\right)-b\left(v_{i-1}\right)}{u_{i}-\gamma\left(v_{i-1}\right)},
$$

holds with $0<\alpha<1$ ( $\alpha$ close to 1 ). The function $v_{i}$ is obtained by the "algebraic correction"

$$
b\left(v_{i}\right):=b\left(v_{i-1}\right)+\mu_{i}\left(u_{i}-\gamma\left(v_{i-1}\right)\right) .
$$

The suggested approximation is based on special semidiscretization in time (see also [9,17-21,24]) which reduces (1.1) to a succession of linear elliptic equations coupled with algebraic correction due to nonlinearity. The nonlinearity of equation is treated by "optimal choice" of special relaxation function corresponding to $\partial_{s}\left(b \circ \gamma^{-1}\right)$, constructed in an iterative way.

Remark. The scheme (1.10)-(1.12) used here is nothing else than "copy-paste" of the one (2.8)-(2.10) from [25]. However, the form (1.10)-(1.12) naturally respects the double-nonlinear structure of (1.1). In this sense, it is close also to approximation suggested in [20]. There, double-nonlinear degenerate parabolic problems are studied, but the blow-up phenomenon cannot be included in the framework.

The scheme (1.10)-(1.12) is linear but not explicit due to $u_{i}, \mu_{i}$. The question is how to find in a constructive way this couple simultaneously satisfying (1.10)-(1.11). From the background of 
the method, reaching realistic and precise numerical results requires to force the relaxation function very near to the difference quotient on the right hand side of (1.11). It corresponds to the value of $b^{\prime}(\xi) / \gamma^{\prime}(\xi)$, where $\xi \in\left(v_{i-1}, v_{i}\right)$. To determine $\mu_{i}, u_{i}$ in that way, we use iterations

$$
\begin{aligned}
& \left(\mu_{i, k-1}\left(u_{i, k}-\gamma\left(v_{i-1}\right)\right), \varphi\right)+\tau\left(\partial_{x} \widetilde{\beta}\left(x, u_{i, k}\right), \varphi_{x}\right)=\tau\left(\widetilde{\beta}\left(x, u_{i, k}\right), \varphi\right), \\
& \bar{\mu}_{i, k}=\frac{b\left(\gamma^{-1}\left(\alpha u_{i, k}+(1-\alpha) \gamma\left(v_{i-1}\right)\right)\right)-b\left(v_{i-1}\right)}{u_{i, k}-\gamma\left(v_{i-1}\right)}, \\
& \mu_{i, k}:=\bar{\mu}_{i, k}, \quad \text { for } 1 \leqslant k \leqslant k_{0}, \quad \mu_{i, k}:=\min \left\{\bar{\mu}_{i, k}, \mu_{i, k-1}\right\}, \quad \text { for } k=k_{0}+1, \ldots,
\end{aligned}
$$

starting with

$$
\mu_{i, 0}=b^{\prime}\left(v_{i-1}\right) / \gamma^{\prime}\left(v_{i-1}\right) .
$$

By construction (1.14) with $k_{0} \geqslant 1$, the sequence $\left\{\mu_{i, k}\right\}$ is forced to be monotone and hence convergent. In practical implementations $k_{0}$ can be chosen in accordance with the shape of $b$ and $\beta$ (e.g., sufficiently large, if numerical convergence is observed as in our case). The iterations (1.13)(1.15) converge to functions $u_{i}$ and $\mu_{i}$ satisfying simultaneously (1.10)-(1.11) (see [25, Theorem 2.5]). In practice this convergence is very fast. Practical realization of "algebraic correction" in our case of nonlinear curve shortening is simple, because the inverse function $b^{-1}(s)$ can be determined explicitly. After time discretization, in each iteration, the scheme requires to solve the linear convection-diffusion equation (with convective term only in the presence of anisotropy). For this purpose we use the socalled "power-law scheme" described in [27], which respects the "up-wind" principle.

From $v_{i}, u_{i}$ obtained in each time step of (1.10)-(1.12), the Rothe functions

$$
\begin{array}{lll}
\bar{v}^{(n)}(t)=v_{i}, & \text { for } t_{i-1}<t \leqslant t_{i}, i=1, \ldots, n, & \bar{v}^{(n)}(0)=v_{0}, \\
\bar{u}^{n}(t)=u_{i}, & \text { for } t_{i-1}<t \leqslant t_{i}, i=1, \ldots, n, & \bar{u}^{n}(0)=u_{0}
\end{array}
$$

are constructed. Then due to [25, Theorems 2.3 and 2.4] we have

$$
\bar{v}^{(n)} \rightarrow v \quad \text { in } L_{2}\left(Q_{T}\right), \quad \bar{u}^{n} \rightarrow \gamma(v) \quad \text { in } L_{2}(I, V),
$$

where $v$ is a unique bounded weak solution of the problem (1.1).

\section{Discussion on numerical computations}

This section is devoted to the presentation of the numerical results obtained by approximation scheme (1.10)-(1.12) in solving problem (1.1) in the case of anisotropic nonlinear curvature driven evolution (1.2). In each time step we use a few iterations (1.13)-(1.15) to find a couple $u_{i}, \mu_{i}$ satisfying the convergence condition (1.11). After that, using algebraic correction (1.12), we obtain the function $v_{i}$, which corresponds to the unknown curvature $k(\theta, t)$ in time instant $t_{i}$.

Below, we document graphically the computed results. In plotting time moments, the curves are reconstructed from the computed curvature function by the formula (1.3); the integral there is evaluated numerically. Because the curves are given by (1.3) uniquely up to a translation, for determining the real evolution we need to know the motion of at least one point of the curve. For this purpose we use (1.2) or some other criterion, e.g., conservation of the center of the mass. The numerical flow of 
curves computed by our technique (which is not based on computing the position vector) satisfies the closeness condition

$$
\int_{0}^{2 \pi \nu} \frac{\mathrm{e}^{\mathrm{i} \theta}}{k(\theta, t)} \mathrm{d} \theta=0
$$

very precisely in all presented cases. Thus no curve on figures is split. This rather surprising property of the scheme is an indicator of its correctness and effectiveness.

For simplicity, in the following lines, we will write only $\beta(k)$ in the cases without anisotropy. It is an easy computation that a special solution of (1.2) with $\beta(k)=k^{m}, m>0$, is a circle shrinking in selfsimilar form to the center; its radius $R(t)$ is given by

$$
R(t)=\left(R(0)^{m+1}-(m+1) t\right)^{1 /(m+1)} .
$$

From this formula we obtain exact blow-up time for curvature. Table 1 expresses the relation between exact and numerically computed blow-up times. For the power $m$ in the function $\beta$, we list the exact blow-up time $T$, and numerically computed ones for $\tau=0.01, \tau=0.001$, respectively. The written equidistant time step is used in computations until a time when the curvature starts to growth very rapidly (e.g., for $m=1 / 3$ we use $\tau=0.01$ up to 0.73 and $\tau=0.001$ up to 0.748 , respectively) and then, we adaptively refine the time step to obtain numerical blow-up (curvature of order $10^{3}$ ).

In Figs. 3-8, we plot the evolution of the initial ellipse, with halfaxes $a=2, b=1$, governed by (1.2), with several choices of $\beta$.

Fig. 3 represents the classical linear curve shortening, when the circle is asymptotical shape of evolution [14].

The next figure represents the behaviour for $\beta(k)=k^{m}, m>1$; the asymptotical motion is similar to the previous one, the extinction is faster and the "slow diffusion effect" known from solving the

Table 1

\begin{tabular}{lllllll}
\hline$m$ & $1 / 10$ & $1 / 4$ & $1 / 3$ & $1 / 2$ & 2 & 4 \\
\hline$T$-exact & $0.90 \overline{90}$ & 0.8 & 0.75 & $0.6 \overline{6}$ & $0.3 \overline{3}$ & 0.2 \\
$\tau=0.01$ & 0.9069 & 0.7959 & 0.7449 & 0.6592 & 0.3185 & 0.1825 \\
$\tau=0.001$ & 0.9084 & 0.7994 & 0.7493 & 0.6659 & 0.3315 & 0.1981 \\
\hline
\end{tabular}

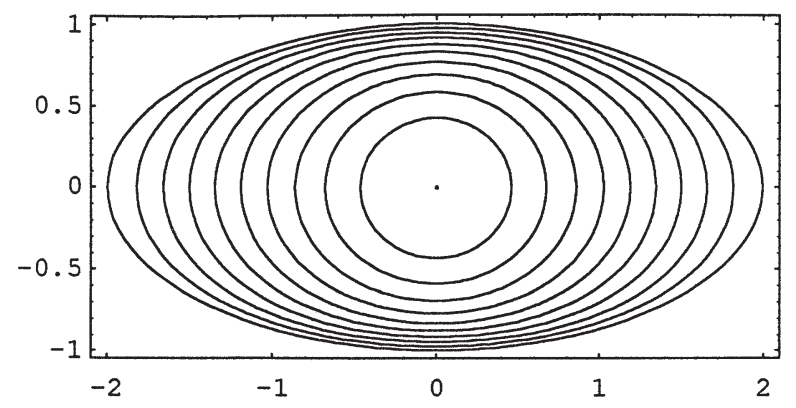

Fig. 3. $\beta(k)=k$, the numerical blow-up $T=1.0006$, plotting step $\tau_{g}=0.1$. 


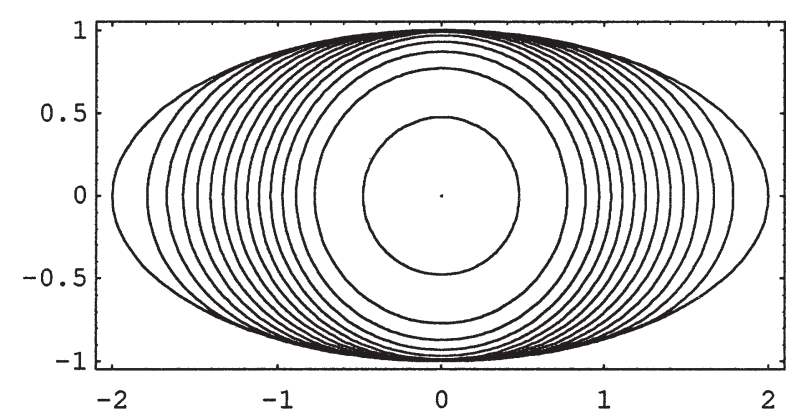

Fig. 4. $\beta(k)=k^{4}$, the numerical blow-up $T=0.713$, plotting step $\tau_{g}=0.05$.

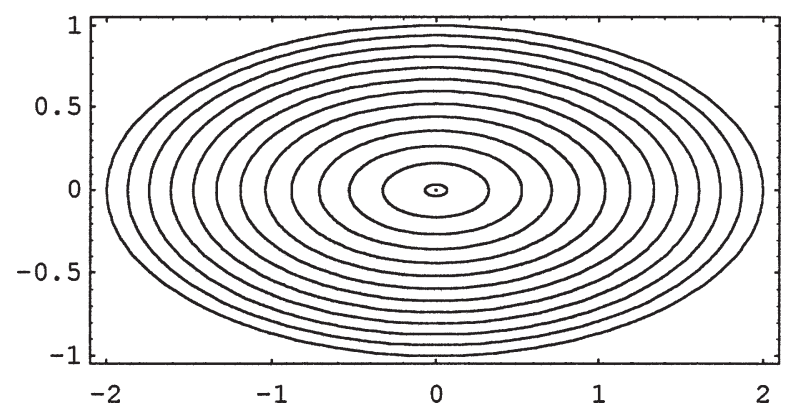

Fig. 5. $\beta(k)=k^{1 / 3}$, the numerical blow-up $T=1.1908$, plotting step $\tau_{g}=0.1$.

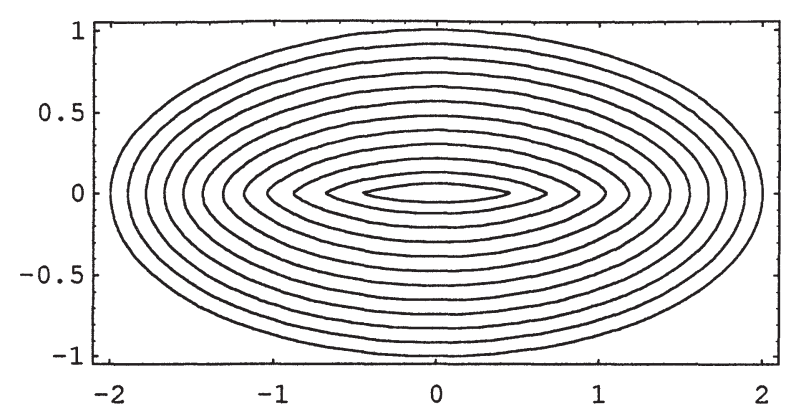

Fig. 6. $\beta(k)=k^{1 / 10}$, the numerical blow-up $T>1.054$, plotting step $\tau_{g}=0.1$ and 1.054 .

porous-medium equation is expressed here in a very slow motion of the points in which the curvature is close to 0 .

In the special case $\beta(k)=k^{1 / 3}$ (Fig. 5), the arbitrary ellipse is a selfsimilar solution (see $[2,28]$ ). This phenomenon is respected also in our numerical computations. We check it by time evolution of the ratio $a / b$ on which there are practically no changes up to times very near to shrinking, and also by the time evolution of the isoperimetric ratio

$$
\text { Iso }=\frac{L}{4 \pi S}
$$




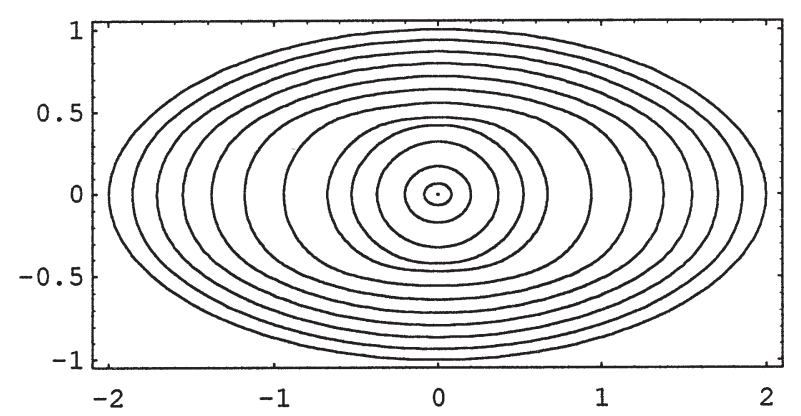

Fig. 7. $\beta(k)$-combination of $k^{1 / 2}, k^{2}, k^{1 / 3}$, the numerical blow-up $T=0.895$, plotting step $\tau_{g}=0.1$ up to 0.7 and then $0.75,0.85,0.88,0.895$.

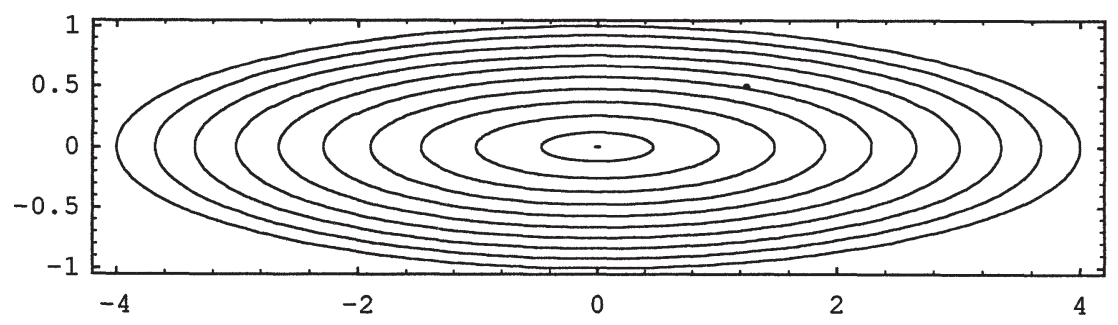

Fig. 8. $\beta(k)=k^{1 / 3}$, the numerical blow-up $T=1.8917$, plotting step $\tau_{g}=0.2$.

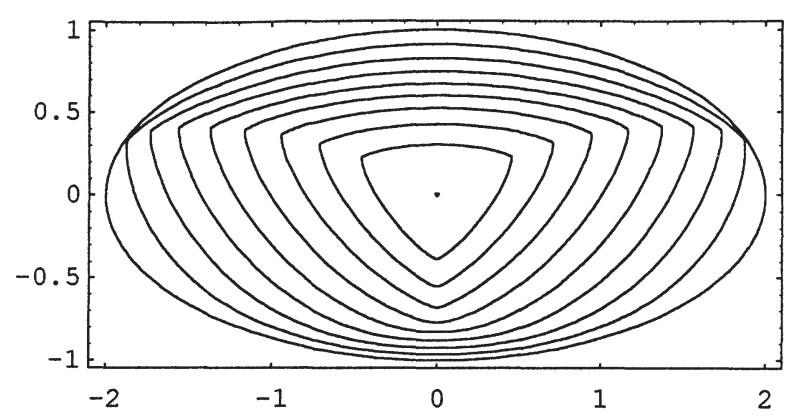

Fig. 9. $\beta(\theta, k)=(1-(8 / 9) \cos \theta) k^{1 / 3}$, the numerical blow-up $T=0.9398$, plotting step $\tau_{g}=0.1$.

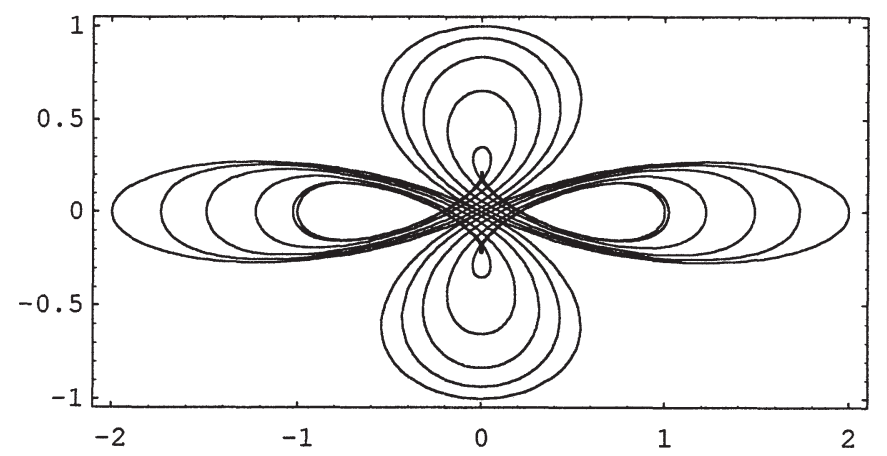

Fig. 10. $\beta(k)=k$. 


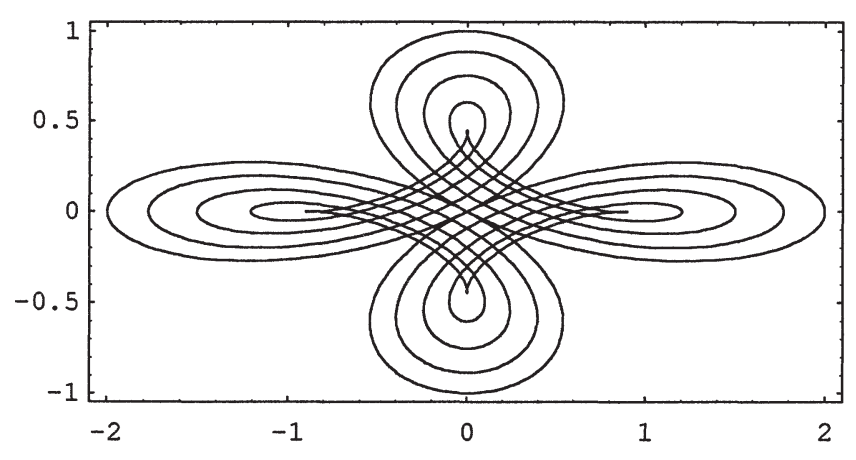

Fig. 11. $\beta(k)=k^{1 / 3}$.

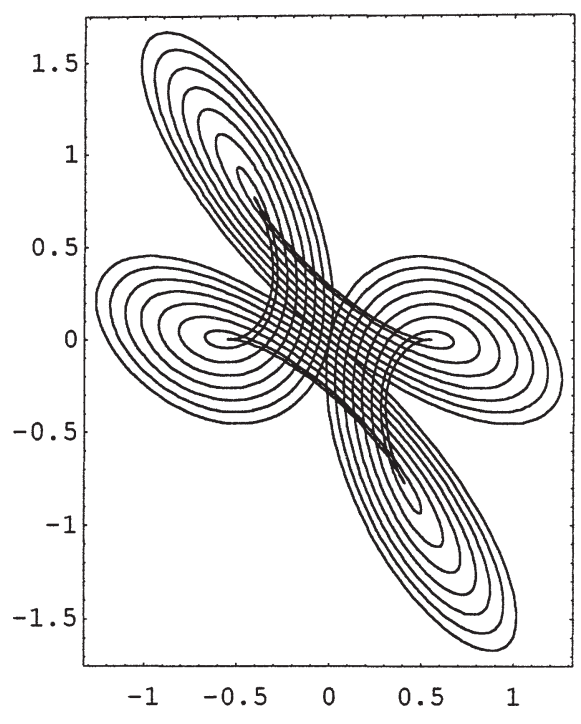

Fig. 12. $\beta(k)=k^{1 / 3}$.

( $L$-length of the curve, $S$-enclosed area) computed for evolving shapes. It is invariant for the numerical solution, too. The conservation of the previous two quantities is observed also in the more complicated experiment $(a / b=4)$ documented in Fig. 8. For those experiments, the exact blow-up time can be computed [26] and is equal to $(3 / 4)(a b)^{2 / 3}$. So in the first case the exact one is equal 1.19055 while the numerically computed is 1.1908 ; in the second case we have 1.88988 and 1.8917 , respectively.

Choosing $\beta(k)=k^{1 / m}$, with $m$ large, the motion of points with small curvature is fast and we obtain behaviour as in Fig. 6.

In Fig. 7 the function $\beta$ is given as a joining of $k^{1 / 2}, k^{2}, k^{1 / 3}$ functions in intervals $[0,1),[1,2)$, $[2, \infty)$, respectively. (Note that such $\beta$ is a little bit worse than assumed in $(\mathrm{H} 3)-(\mathrm{H} 4)$.) In this case, we see several changes in the evolving shape and, only after that, shrinking to the center in ellipse-like form.

Fig. 9 represents an anisotropic version of the evolution from Fig. $5, \beta(\theta, k)=(1-(8 / 9) \cos \theta) k^{1 / 3}$. 


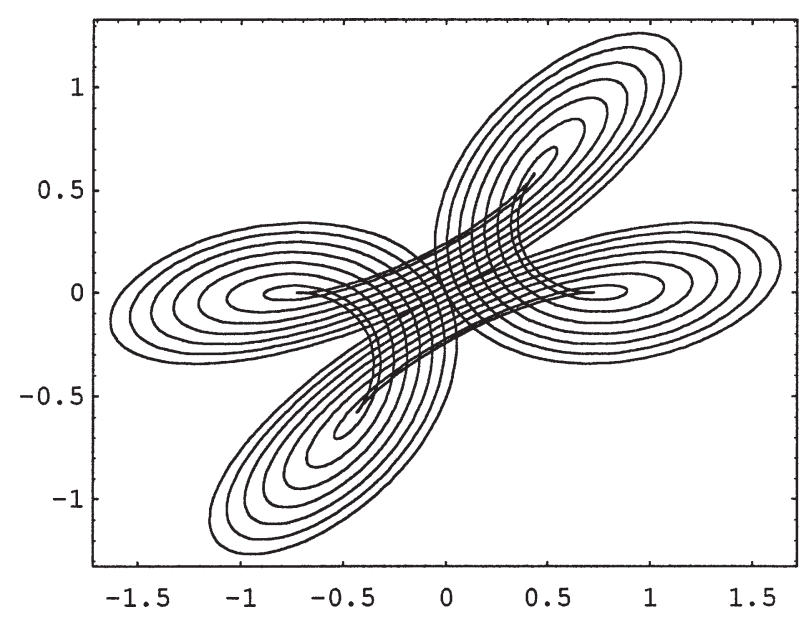

Fig. 13. $\beta(k)=k^{1 / 3}$.

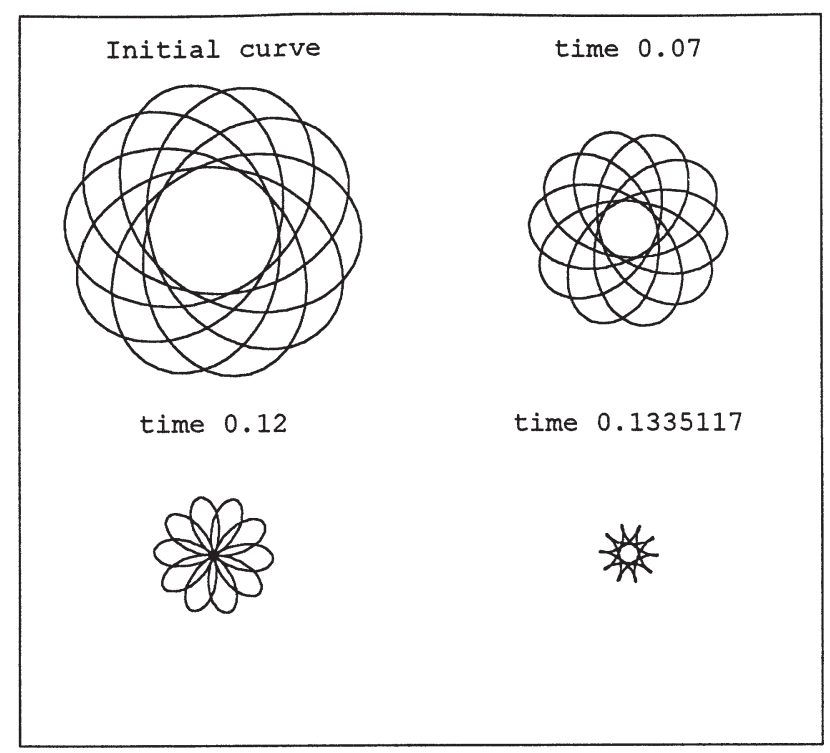

Fig. 14. $\beta(k)=k^{1 / 3}$.

In all previous graphically documented numerical experiments (shrinking of ellipses) the time step $\tau=0.001$ (in the sense of description above Table 1), the space discretization step $h=2 \pi / 200$, and in the labels of figures we print the form of $\beta$, numerical blow-up time $T$ and time steps in which the solutions are plotted.

If the index $\nu$ of an initial closed convex curve is greater than 1, then the corresponding solution can become singular without shrinking to a point. Such process for linear curve shortening flow was studied in [5] and from a numerical point of view in [25]. 


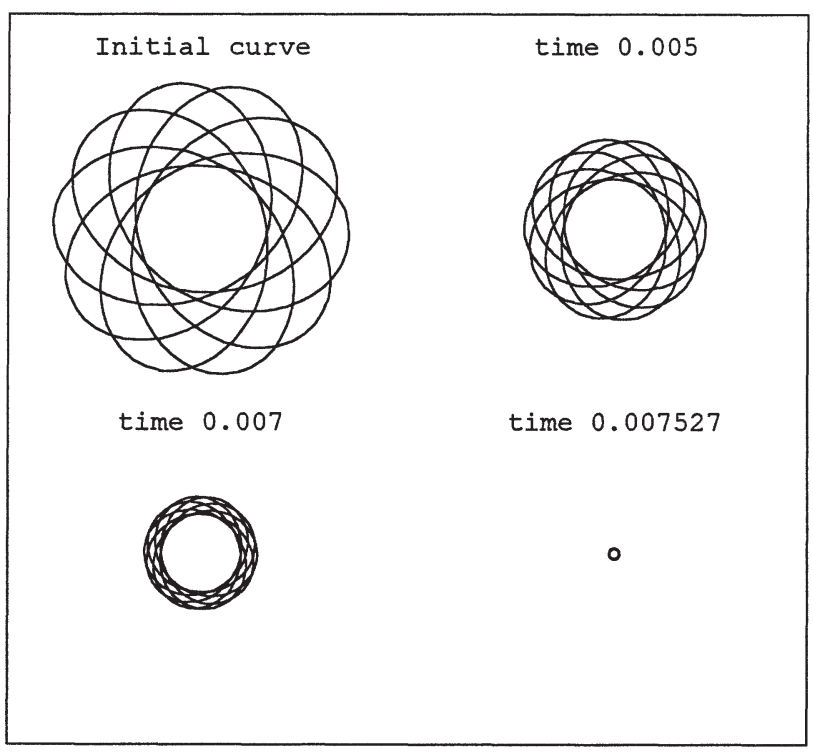

Fig. 15. $\beta(k)=k^{2}$.

Symmetric shape, like 4-petal, forms four singularities at the same time (see also [25, Fig. 6a]) for any choice of $\beta(k)$. If we apply affine transformation on that initial curve and then use the linear model for motion, less than four singularities are formed finally at the same time-see Fig. 10.

In spite of this, if we choose $\beta(k)=k^{1 / 3}$, the behaviour of the affine transformed initial curve corresponds to the behaviour of the original 4-petal. Figs. 11-13 illustrate the fact that the blowup of the curvature in four subintervals of $\Omega$ occurs at the same time again. This phenomenon is an interesting demonstration of the affine invariantness of the Alvarez, Guichard, Lions and Morell model of multiscale shape analysis.

From the linear isotropic curve shortening, the existence of selfintersecting selfsimilar solutions, Abresch-Langer functions, is known. The evolution of one of them for $\beta(k)=k^{1 / 3}$ and $\beta(k)=k^{2}$, respectively, is documented in Figs. 14 and 15. The formation of singularities in the first case and rounding the circle and then shrinking to the point in the second one were computed (for selfsimilar motion see [25, Fig. 8d]).

The interesting open question is how to use some modification of the computational method presented here for computing the general nonconvex curve evolution. The other successful technique [12] for computing linear curve shortening flow can be, at least from an implementation point of view, also adapted to solve (1.2) [26].

\section{Acknowledgements}

I would like to thank to Professors W. Jäger and G. Dziuk for their interest in my work and the invitation for a short stay at the Universities of Heidelberg and Freiburg, where my attention has been attracted to the nonlinear version of curve shortening flow and its usage in image processing. 


\section{References}

[1] U. Abresch and J. Langer, The normalized curve shortening flow and homothetic solutions, J. Differential Geom. 23 (1986) 175-196.

[2] L. Alvarez, F. Guichard, P.L. Lions and J.M. Morel, Axioms and fundamental equations of image processing, Arch. Rational Mech. Anal. 123 (1993) 200-257.

[3] L. Alvarez, P.L. Lions and J.M. Morel, Image selective smoothing and edge detection by nonlinear diffusion II, SIAM J. Numer. Anal. 29 (1992) 845-866.

[4] L. Alvarez and J.M. Morel, Formalization and computational aspects of image analysis, Acta Numerica (1994) 1-59.

[5] S.B. Angenent, On the formation of singularities in the curve shortening flow, J. Differential Geom. 33 (1991) 601-633.

[6] S.B. Angenent, Parabolic equations for curves on surfaces (parts I and II), Ann. Math. 133 (1991) 171-215.

[7] S.B. Angenent and M.E. Gurtin, Multiphase thermomechanics with an interfacial structure 2. Evolution of an isothermal interface, Arch. Rational Mech. Anal. 108 (1989) 323-391.

[8] S.B. Angenent and M.E. Gurtin, Anisotropic motion of a phase interface. Well-posedness of the initial value problem and qualitative properties of the interface, J. Reine Angew. Math. 446 (1994) 1-47.

[9] A.E. Berger, H. Brezis and J.C.W. Rogers, A numerical method for solving the problem $u_{t}-\Delta f(u)=0$, RAIRO Anal. Numer. 13 (1979) 297-312.

[10] Y.-G. Chen, Y. Giga and S. Goto, Uniqueness and existence of viscosity solutions of generalized mean curvature flow equation, J. Differential Geom. 33 (1991) 749-786.

[11] M.G. Crandall, H. Ishii and P.L. Lions, User's guide to viscosity solutions of second order partial differential equations, Bull. Amer. Math. Soc. (N.S.) 27 (1992) 1-67.

[12] G. Dziuk, Convergence of a semi discrete scheme for the curve shortening flow, Math. Models Methods Appl. Sci. 4 (1994) 589-606.

[13] L.C. Evans and J. Spruck, Motion of level sets by mean curvature I, J. Differential Geom. 33 (1991) 635-681.

[14] M. Gage and R.S. Hamilton, The heat equation shrinking convex plane curves, J. Differential Geom. 23 (1986) 285-314.

[15] M. Grayson, The heat equation shrinks embedded plane curves to round points, J. Differential Geom. 26 (1987) 285-314.

[16] A. Handlovičová and K. Mikula, Numerical solution of parabolic equations related to level set formulation of mean curvature flow, to appear.

[17] W. Jäger and J. Kačur, Solution of porous medium type systems by linear approximation schemes, Numer. Math. 60 (1991) 407-427.

[18] W. Jäger and J. Kačur, Approximation of degenerate elliptic-parabolic problems by nondegenerate elliptic and parabolic problems, Preprint, University of Heidelberg (1991).

[19] W. Jäger and J. Kačur, Solution of doubly nonlinear and degenerate parabolic problems by relaxation schemes, $M^{2} A N 29$ (1995) 605-627.

[20] J. Kačur, Solution of some free boundary problems by relaxation schemes, Preprint No. M3-94, Faculty of Mathematics and Physics, Comenius University Bratislava (1994).

[21] J. Kačur, A. Handlovičová and M. Kačurová, Solution of nonlinear diffusion problems by linear approximation schemes, SIAM J. Numer. Anal. 30 (1993) 1703-1722.

[22] J. Kačur and K. Mikula, Solution of nonlinear diffusion appearing in image smoothing and edge detection, Appl. Numer. Math. 17 (1995) 47-59.

[23] P.L. Lions, Axiomatic derivation of image processing models, Math. Models Methods Appl. Sci. 4 (1994) 467-475. 
[24] E. Magenes, R.H. Nochetto and C. Verdi, Energy error estimates for a linear scheme to approximate nonlinear parabolic problems, Math. Mod. Numer. Anal. 21 (1987) 655-678.

[25] K. Mikula and J. Kačur, Evolution of convex plane curves describing anisotropic motions of phase interfaces, SIAM J. Sci. Comput. 17 (1996).

[26] K. Mikula and D. Ševčovič, Approximation scheme for nonlinear curve shortening flow, in preparation.

[27] S. Patankar, Numerical Heat Transfer and Fluid Flow (Hemisphere Publ. Corp., New York, 1980).

[28] G. Sapiro and A. Tannenbaum, Affine shortening of non-convex plane curves, Preprint, Dept. of Electrical Engineering, Technion, Israel Institute of Technology, Haifa (1992).

[29] A. Schmidt, Die Berechnung Dreidimensionaler Dendriten mit Finiten Elementen, Ph.D. thesis, Freiburg University (1993). 